\title{
Minimality of State Space Solutions of DSGE Models and Existence Conditions for Their VAR Representation
}

\author{
Massimo Franchi · Paolo Paruolo
}

Accepted: 13 August 2014 / Published online: 12 October 2014

(C) European Union 2014

\begin{abstract}
Standard solution methods of DSGE models do not necessarily deliver minimal state space forms. When the ABCD form is non-minimal, the conditions in the literature are not necessary for the existence of a VAR representation of the observables. In this paper we present necessary and sufficient conditions that are valid in general, and hence can be applied to minimal and non-minimal ABCD forms. If the state space form is minimal, our conditions coincide with those in the literature. These results also clarify that it is possible to have unstable eigenvalues together with a (possibly finite) VAR representation of the observables.
\end{abstract}

Keywords DGSE models · VAR models · Fundamentalness · Finite order VAR . Minimal state space representation

JEL Classification $\quad \mathrm{C} 18 \cdot \mathrm{C} 32 \cdot \mathrm{E} 32$

\section{Introduction}

Economic shocks of DSGE models cannot always be recovered from VARs. This situation has been discussed e.g. in Chari et al. (2005), Christiano et al. (2006), Kapetanios

Electronic supplementary material The online version of this article (doi:10.1007/s10614-014-9465-4) contains supplementary material, which is available to authorized users.

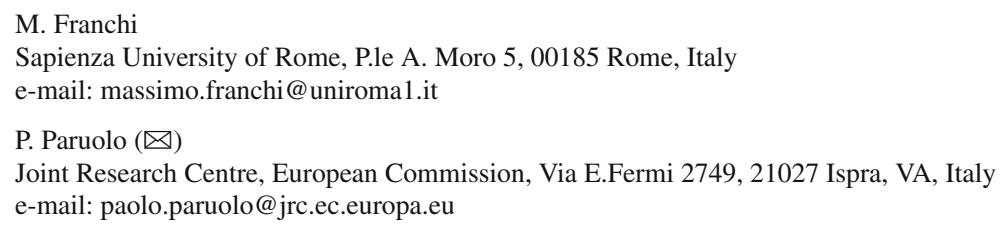


et al. (2007), Ravenna (2007), and it is related to the non-fundamentalness of economic models, see Hansen and Sargent (1980), Lippi and Reichlin (1993, 1994) for early treatments of the problem.

Solutions of linear (or linearized) DSGE models can be expressed in linear state space form using several solution methods, such as Binder and Pesaran (1997), Uhlig (1999), Klein (2000), Sims (2001). In the following, we refer to the linear state space form as the $\mathrm{ABCD}$ form. When the number of shocks is equal to the number of observables and the matrix that loads the former into the latter is non-singular, the $\mathrm{ABCD}$ form is called square. We observe that the ABCD form of the solution dynamics need not be minimal, i.e. it may involve a non-minimal number of state variables. This is illustrated for example in Komunjer and $\mathrm{Ng}$ (2011), who show that the state space solutions in Christiano et al. (2005), An and Schorfheide (2007), Smets and Wouters (2007), García-Cicco et al. (2010) are all non-minimal.

In this paper we investigate population conditions for fundamentalness of the ABCD form and for the existence of a finite VAR representation of the observables. ${ }^{1}$ Fundamentalness is currently checked using the 'poor man's invertibility condition' in Fernández-Villaverde et al. (2007); applications of this approach can be found in Leeper et al. (2013), Schmitt-Grohé (2010), Kurmann and Otrok (2011), Sims (2012) inter alia. The poor man's invertibility condition consists in the stability of the associate state matrix, $F$ say, i.e. the state matrix in the system in which the role of inputs and outputs is interchanged, see Bart et al. (2008). Conditions for the VAR to be of finite order are given in Ravenna (2007), where a 'unimodularity condition' is proposed as a check. This condition consists in the unimodularity of the matrix polynomial $I-F z$, $z \in \mathbb{C},^{2}$ or equivalently in the nilpotency of $F$, see Franchi and Vidotto (2013).

When the square ABCD form is minimal, we show that the poor man's invertibility condition and the unimodularity condition are proper checks, i.e. they are necessary and sufficient conditions for fundamentalness. When the ABCD form is non-minimal, we find that the poor man's invertibility condition and the unimodularity condition are not necessary. In other words $F$ can be unstable while the square ABCD form is fundamental and $I-F z$ can be non-unimodular while the square ABCD form has a finite order VAR representation.

Because in current economic practice systems are not always in minimal state space form, in this paper we present alternative orthogonality conditions that are necessary and sufficient and which hold in possibly non-minimal square systems. This provides a check that does not require to transform the system to minimal ABCD form. These orthogonality conditions involve the eigenvectors associated with eigenvalues of the associate state matrix $F$, and correspond to the case where there exist states that are cancelled in the VAR representation of the observables. For fundamentalness, the orthogonality conditions must apply to all the unstable eigenvalues, while for the

\footnotetext{
1 Recall that a square ABCD form is called fundamental if there exist a (possibly infinite order) VAR representation for the observables with respect to the economic shocks, see Hansen and Sargent (1980), Brockwell and Davis (1987), Lippi and Reichlin (1993, 1994).

2 A matrix polynomial is called unimodular if its determinant is a nonzero constant, see e.g. Antsaklis and Michel (2007, p. 283).
} 
existence of a finite VAR representation, the orthogonality conditions must hold for every nonzero eigenvalue.

When the orthogonality conditions hold, we find that the eigenvalues of the associate state matrix $F$ are also eigenvalues of the state matrix, $A$ say. This implies that when the eigenvalues of $A$ and $F$ are different, the orthogonality conditions do not hold. This provides a simplified strategy based solely on eigenvalues of $A$ and $F$. Finally we find that if the ABCD form is minimal, the orthogonality conditions coincide with the poor man's invertibility condition and the unimodularity condition. This shows that the present orthogonality conditions are an extension of the conditions in the literature that apply to general (i.e. not necessarily minimal) linear state space forms.

Therefore, invertibility of linearized DSGE models should be checked either using the present orthogonality conditions or by first transforming the state space form to a minimal representation and then by applying the poor man's invertibility condition to the minimal state space form. Similarly, existence of a finite VAR representation should be checked either using the present orthogonality conditions or by first transforming the state space form to a minimal representation and then by applying the unimodularity condition to the minimal state space form, see Franchi (2013) for an illustration on the Smets and Wouters (2007) model.

The results of the paper employ well-known concepts from systems theory, see e.g. Kailath (1980), Antsaklis and Michel (2007). The possibility to have cancellations of the type discussed in this paper in non-minimal state space systems was noticed by Rosenbrock (1970) inter alia, see his remark after Theorem 1.2, page 38 and Corollary 3 , page 115 . Here we give explicit algebraic condition on the system matrices that characterizes this situation. These tools are meant to be used by macro-economists, who deal with non-minimal state space representations of linearized DGSE models in practice and who are interested in determining if the observables in their model admit a VAR representation.

The results in this paper are also related to cointegration. ${ }^{3}$ The possibility to have unstable states within a non-minimal state space gives in fact one more way to incorporate cointegration in DGSE models, by allowing unit-root stochastic trends in the states that can be canceled in the stable VAR representation of observables.

The rest of the paper is organized as follows. Section 2 introduces the square case. Section 3 shows that fundamentalness and the existence of a finite order VAR can be characterized in terms of an appropriate transfer function. Section 4 presents the orthogonality conditions. Section 5 specializes results to minimal square ABCD forms, showing that for minimal state spaces the orthogonality conditions coincide with the poor man's invertibility condition and the unimodularity condition. Section 6 concludes. All proofs are collected in the Appendix.

\footnotetext{
3 Inversions of matrix polynomials are used in the representation theory for non-stationary processes developed in Johansen (1988, 1996, 2009); see also Franchi and Paruolo (2011a,b). Howlett (1982) focuses on a similar problem in the context of input retrieval for linear systems.
} 


\section{Square ABCD Forms}

Consider an equilibrium dynamics of a linearized DSGE model with representation

$$
\begin{aligned}
& x_{t}=A x_{t-1}+B w_{t} \\
& y_{t}=C x_{t-1}+D w_{t}
\end{aligned}
$$

where $x_{t}$ has dimension $n_{x} \times 1, y_{t}$ has dimension $n_{y} \times 1$ and $w_{t}$ is a white noise of dimension $n_{w} \times 1$ with identity covariance matrix. $A$ is called the state matrix and we refer to (1) as the ABCD form.

The following assumption is maintained throughout the paper.

Assumption 2.1 (Square $A B C D$ form). The matrix $D$ is square and invertible.

In square $\mathrm{ABCD}$ forms the number of economic shocks is equal to the number of observables and the matrix that loads the shocks into the observables is non-singular. In this case one can substitute $w_{t}=D^{-1}\left(y_{t}-C x_{t-1}\right)$ in the first equation in (1) and rewrite the square $\mathrm{ABCD}$ form in the 'associate state space form', see e.g. Ch.2.2 in Bart et al. (2008),

$$
\begin{aligned}
x_{t} & =F x_{t-1}+B D^{-1} y_{t}, \quad F=A-B D^{-1} C, \\
w_{t} & =-D^{-1} C x_{t-1}+D^{-1} y_{t},
\end{aligned}
$$

where the role of input and output of system (1) are interchanged; $F$ is called the 'associate state matrix'.

The ABCD form is called minimal, see e.g. Ch.6 in Kailath (1980) or Ch.2 in Hannan and Deistler (1988), if the dimension of $x_{t}$ is as small as possibile. Minimality holds if and only if the ABCD form is both controllable and observable, i.e. if rank $\mathcal{C}=$ $\operatorname{rank} \mathcal{O}=n_{x}$, where

$$
\mathcal{C}=\left(\begin{array}{llll}
B & A B & \cdots & A^{n_{x}-1} B
\end{array}\right), \quad \mathcal{O}=\left(\begin{array}{c}
C \\
C A \\
\vdots \\
C A^{n_{x}-1}
\end{array}\right)
$$

are respectively called controllability and observability matrix.

Some remarks are in order.

Remark 2.2 The state matrix $A$ may be stable or unstable. ${ }^{4}$ Cases with unstable $A$ are interesting from an economic point of view because they arise from models with unit roots in the endogenous state variables as well as those with non-stationary exogenous shocks, as in Chang et al. (2007).

\footnotetext{
4 A square matrix is called stable when all its eigenvalues are stable, i.e. of modulus strictly less than one; if it has unstable eigenvalues then it is called unstable.
} 
Remark 2.3 The ABCD form may be minimal or non-minimal. Non-minimal ABCD forms are natural outcomes of standard solution methods: for example, Komunjer and $\mathrm{Ng}$ (2011) show that, using gensys and dynare, the ABCD forms in Christiano et al. (2005), An and Schorfheide (2007), Smets and Wouters (2007), García-Cicco et al. (2010) are all non-minimal.

\section{Transfer Function}

In square ABCD forms, it is of interest, see e.g. Fernández-Villaverde et al. (2007), to characterize situations in which the economic shocks can be recovered from the infinite history of the observable variables, i.e. cases in which $y_{t}$ admits an infinite order VAR representation

$$
A(L) y_{t}=u_{t}, \quad A(z)=I_{n_{y}}-\sum_{j=1}^{\infty} A_{j} z^{j}, \quad u_{t}=M w_{t}
$$

where the sequence $\left\{A_{j}\right\}_{j=1}^{\infty}$ is absolutely summable and $M$ is a non-singular matrix that links economic shocks and reduced form errors. When (4) holds, (1) is said to be invertible (and $w_{t}$ fundamental), see Hansen and Sargent (1980), Brockwell and Davis (1987), Lippi and Reichlin (1993, 1994). Furthermore it is of interest, see e.g. Ravenna (2007), to have conditions that describe when the VAR representation of $y_{t}$ has finite order, i.e. when $A(z)$ is a matrix polynomial.

In this section we link the existence of a (possibly finite) VAR representation of $y_{t}$ to properties of the transfer function from $y_{t}$ to $D w_{t}, T(L) y_{t}=D w_{t}$, implied by the associate state space form (2), where

$$
T(z)=I_{n_{y}}-C\left(I_{n_{x}}-F z\right)^{-1} B D^{-1} z, \quad z \in \mathbb{C} .
$$

The relationship between the VAR representation of $y_{t}$ and the transfer function $T(z)$ is presented next; in the following we say that a function is regular if it is finite on the unit disc, i.e. for all $z \in \mathbb{C}$ such that $|z|<1+\delta$, for some $\delta>0$, see Johansen (2009) for a similar notion of regularity. Remark that the coefficients of the expansion of a regular function $f(z)=\sum_{j=0}^{\infty} f_{j} z^{j}$ are geometrically decreasing and hence absolutely summable.

Proposition 3.1 (Transfer function conditions). The vector of observables $y_{t}$ admits an infinite order VAR representation if and only if $T(z)$ is regular. Moreover, the VAR representation is of finite order if and only if $T(z)$ is a matrix polynomial. In either case, the coefficients of the VAR representation of $y_{t}$ and the mapping between economic shocks and reduced form errors in (4) are given by $A(z)=T(z)$ and $M=D$.

The properties of the matrix function $T(z)$ depend on those of $(I-F z)^{-1}=$ $\operatorname{adj}(I-F z) /|I-F z|$. The roots of $|I-F z|=0$ are poles of $(I-F z)^{-1}$; because $|I-F z|=0$ if and only if $z=\lambda_{u}^{-1}$, where $\left\{\lambda_{u}\right\}$ are the nonzero eigenvalues of $F$, if $F$ is stable then $(I-F z)^{-1}$ is regular because $\min _{u}\left|\lambda_{u}^{-1}\right|>1$. Hence $T(z)$ 
is regular and, by Proposition 3.1, this implies that $y_{t}$ admits an infinite order VAR representation. Moreover, if $F$ is nilpotent then $I-F z$ is unimodular and $(I-F z)^{-1}$ is a matrix polynomial; hence $T(z)$ is a matrix polynomial and, by Proposition 3.1, this implies that $y_{t}$ admits a finite order VAR representation.

Observe that $T(z)$ can be regular even if $F$ is unstable and it can be a matrix polynomial even if $F$ is not nilpotent. In fact, when $F$ is unstable, $(I-F z)^{-1}$ has poles in the (closed) unit disc and thus it is non-regular, but those singularities may be absent from $C(I-F z)^{-1} B$ due to the presence of $B$ and $C$. In this case $C(I-F z)^{-1} B$ is regular and thus the same holds for $T(z)$. This possibility is illustrated in the following example, which serves as a running example in the rest of the paper.

Example 3.2 (Running example). This is a special case of the model in Chang et al. (2007). Let $n_{x}=4, n_{w}=n_{y}=2$ and

$$
A=\left(\begin{array}{cc}
\Phi_{1} & 0 \\
0 & I_{2}
\end{array}\right), \quad B=\left(\begin{array}{c}
\frac{1}{2} I_{2} \\
I_{2}
\end{array}\right), \quad C=\left(\Phi_{1} \Phi_{2}\right), \quad D=\Phi_{2}+\frac{1}{2} I_{2}
$$

where

$$
\Phi_{1}=\left(\begin{array}{cc}
1 & 1 \\
-\frac{1}{2} & -\frac{1}{2}
\end{array}\right), \quad \Phi_{2}=\left(\begin{array}{ll}
1 & 1 \\
1 & 1
\end{array}\right)
$$

Observe that $D$ is non-singular so that the $\mathrm{ABCD}$ form is square. Moreover $F=$ $A-B D^{-1} C$ has eigenvalues $\{1,3 / 5,0,0\}$, and $(I-F z)^{-1}$ has a pole at $z=1$ and thus it is non-regular. At the same time $T(z)$ is regular, because

$$
C\left(I_{n_{x}}-F z\right)^{-1} B=\frac{5}{4(3 z-5)}\left(\begin{array}{cc}
4 z-6 & 4 z-6 \\
z-3 & z-3
\end{array}\right)
$$

has no pole at $z=1$ (it only has a pole at $z=\frac{5}{3}$ ) and hence the expansion $T(z)=$ $\sum_{j=0}^{\infty} T_{j} z^{j}$ has absolutely summable coefficients. This illustrates that one can have fundamentalness together with unstable $F$.

Similarly, one can construct examples in which $B$ and $C$ cancel every pole of $(I-F z)^{-1}$ so that $C(I-F z)^{-1} B$ is a matrix polynomial, which in turn implies that the same holds for $T(z)$. This shows that the poor man's invertibility condition in Fernández-Villaverde et al. (2007) and the unimodularity condition in Ravenna (2007) are not necessary conditions for a VAR representation of a square ABCD form. The next proposition proves that they are sufficient conditions.

Proposition 3.3 (Sufficient conditions). If $F$ is stable, then $y_{t}$ admits an infinite order $V A R$ representation; the converse does not hold. If $F$ is nilpotent, then $y_{t}$ admits a finite order VAR representation; the converse does not hold. 


\section{Conditions for the Existence of a VAR Representation}

In this section we state necessary and sufficient conditions for fundamentalness and for the existence of a finite order VAR, in the form of orthogonality conditions or rank conditions. These conditions apply both to minimal and non-minimal square ABCD forms, and they are based on the idea of pole cancellations in $C(I-F z)^{-1} B$ in the matrix function $T(z)$.

We introduce the following notation: let $\lambda_{u}, u=1, \ldots, q$, be all the distinct nonzero eigenvalues of $F$ and apply the partial fraction expansion ${ }^{5}$

$$
\begin{aligned}
& (I-F z)^{-1}=P(z)+H(z), \quad P(z)=\sum_{u=1}^{q} P_{u}(z), \quad P_{u}(z)=\sum_{j=1}^{m_{u}} \frac{P_{\lambda_{u}, m_{u}-j}}{\left(1-\lambda_{u} z\right)^{j}}, \\
& \quad P_{\lambda_{u}, 0} \neq 0 .
\end{aligned}
$$

Here $H(z)$ is a matrix polynomial, $P(z)$ is the sum of the principal parts $P_{u}(z)$ of $(I-F z)^{-1}$ at $z=\lambda_{u}^{-1}$ and $m_{u}$ is the order of the pole of $(I-F z)^{-1}$ at $z=\lambda_{u}^{-1}$. Note that zero eigenvalues of $F$ are absent from $|I-F z|$ and hence they do not appear in the principal part $P(z)$. A characterization of the pole cancellations in $C(I-F z)^{-1} B$ due to the presence of $B$ and $C$ is given in the following Lemma, which define our orthogonality conditions.

Lemma 4.1 (Orthogonality conditions). $T(z)$ has no pole at $z=\lambda_{u}^{-1}$ if and only if the orthogonality conditions

$$
C P_{\lambda_{u}, m_{u}-j} B=0, \quad j=1, \ldots, m_{u}
$$

hold. If the orthogonality conditions hold, then $\lambda_{u}$ is also an eigenvalue of $A$.

Because $P_{u}(z)$ fully characterizes the singularity of $(I-F z)^{-1}$ at $z=\lambda_{u}^{-1}$, in order for $C(I-F z)^{-1} B$ to have no pole at $z=\lambda_{u}^{-1}, B$ and $C$ must be such that $C P_{u}(z) B=0$. This requires each coefficient of the principal part to be 0 when pre and post-multiplied by $C$ and $B$. Lemma 4.1 also states that if a pole cancellation occurs for a given eigenvalue of $F$, then this must also be an eigenvalue of $A$; this observation leads to the following necessary conditions.

Proposition 4.2 (Necessary conditions). The vector of observables $y_{t}$ admits an infinite order VAR representation only if each unstable eigenvalue of $F$ is also an eigenvalue of A. Moreover, the VAR representation is of finite order only if each nonzero eigenvalue of $F$ is also an eigenvalue of $A$.

The main characterization result is stated next.

\footnotetext{
5 See e.g. Fischer and Lieb (2012, Ch. III.2).
} 
Proposition 4.3 (Necessary and sufficient orthogonality conditions). The vector of observables $y_{t}$ admits an infinite order VAR representation if and only if the orthogonality conditions hold at each unstable eigenvalue of F. Moreover, the VAR representation is of finite order if and only if the orthogonality conditions hold at each nonzero eigenvalue of $F$.

An infinite order VAR representation requires the cancellation of all the poles associated with the unstable eigenvalues of $F$; the VAR has finite order when the cancellation also involves the stable nonzero eigenvalues. Note that if all eigenvalues are stable, the first orthogonality condition for invertibility is automatically satisfied because there are no unstable eigenvalues. Similarly, if $F$ is nilpotent, all eigenvalues are equal to 0 , so that $q=0$ in (6) and this implies $P(z)=0$; hence also the second orthogonality condition for the existence of a finite order VAR is automatically satisfied. This illustrates that the poor man's invertibility condition (and the unimodularity condition) implies the orthogonality conditions in Proposition 4.3 but not viceversa. That is, in square $\mathrm{ABCD}$ forms the former is stronger than the latter, and that the conditions differ.

An equivalent and more directly implementable rank condition is provided next. ${ }^{6}$

Proposition 4.4 (Rank conditions). Let $\lambda_{u}$ be a nonzero eigenvalue of $F$, rank decompose $F-\lambda_{u} I=\alpha \beta^{\prime}$, where $\alpha, \beta$ are $n_{x} \times r$ full column rank matrices, and define $\alpha_{\perp}, \beta_{\perp}$ as bases ${ }^{7}$ of the orthogonal complements of the column spaces spanned by $\alpha$ and $\beta$ respectively. Then the orthogonality condition holds at $\lambda_{u}$ if and only if $\operatorname{rank}\left(\alpha_{\perp}^{\prime} B\right)<n_{x}-r$ or $\operatorname{rank}\left(C \beta_{\perp}\right)<n_{x}-r($ or both $)$.

In the light of this equivalence one can thus restate the main characterization result in Proposition 4.3 replacing orthogonality conditions with rank conditions.

Proposition 4.5 (Necessary and sufficient rank conditions). The vector of observables $y_{t}$ admits an infinite order VAR representation if and only if the rank condition holds at each unstable eigenvalue of $F$. Moreover, the VAR representation is of finite order if and only if the rank condition holds at each nonzero eigenvalue of $F$.

A MATLAB script that implements this check is available in the Additional Material to the present paper.

Example 4.6 (Continuation of Example 3.2). We illustrate our results using the running example, where the eigenvalues of $F$ and $A$ are $\sigma_{F}=\{1,3 / 5,0,0\}$ and $\sigma_{A}=\{1,1,1 / 2,0\}$ respectively. Because $3 / 5$ is not an eigenvalue of $A$, the necessary condition in Proposition 4.2 is not satisfied and one immediately sees that $y_{t}$ does not have a finite order VAR representation. Because 1 is also an eigenvalue of $A$, the necessary condition in Proposition 4.2 is satisfied and we proceed to check the rank condition for the eigenvalue equal to 1.

\footnotetext{
${ }^{6}$ Here and in the following $a^{\prime}$ indicates the conjugate transpose of $a$, which reduces to transposition for $a$ real.

7 Hence of full column rank $n_{x}-r$.
} 
Rank-decomposing $F-I=\alpha \beta^{\prime}$, where $\alpha, \beta$ have dimension $4 \times 3$, and defining $\alpha_{\perp}, \beta_{\perp}$ of dimension $4 \times 1$ as bases of the orthogonal complements of $\operatorname{col} \alpha$ and $\operatorname{col} \beta$, one finds $\alpha_{\perp}=(-3:-3: 2: 1)^{\prime}$ and $\beta_{\perp}=(0: 0: 1:-1)^{\prime}$. Because

$$
C=\left(\begin{array}{cccc}
1 & 1 & 1 & 1 \\
-\frac{1}{2} & -\frac{1}{2} & 1 & 1
\end{array}\right)
$$

one has $C \beta_{\perp}=0$ and hence the rank condition in Proposition 4.4 is satisfied at the unstable eigenvalue equal to 1 . This correctly indicates, see Proposition 4.5 , that the vector of observables $y_{t}$ has an infinite order VAR representation.

\section{Minimal Square ABCD Forms}

In this section we treat the special case of square minimal ABCD forms. Minimality could be directly obtained as a property of the DSGE's solution or it could be derived from a non-minimal solution by eliminating irrelevant states, as described e.g. in Rosenbrock (1970). Here we show that if the square ABCD form is minimal, then the orthogonality conditions coincide with the poor man's invertibility condition and with the unimodularity condition. We conclude this section by illustrating the result via the running example.

Proposition 5.1 (Equivalence in minimal state space forms). If the square $A B C D$ form is minimal, then the vector of observables $y_{t}$ admits an infinite order VAR representation if and only if $F$ is stable. Moreover, the VAR representation is of finite order if and only if $F$ is nilpotent.

When the ABCD form is minimal, $C P_{u}(z) B \neq 0$ for any eigenvalue and hence no cancellation is possibile. Then each pole of $(I-F z)^{-1}$ is a pole of $C(I-F z)^{-1} B$ and the orthogonality condition for an infinite order VAR holds (trivially) if and only if $F$ has no unstable eigenvalues. Similarly, it holds (trivially) for a finite order VAR holds if and only if all eigenvalues are equal to zero. This shows that when the square ABCD form is minimal, the poor man's invertibility condition and the unimodularity condition are equivalent to the orthogonality conditions and thus they become necessary and sufficient.

Example 5.2 (Continuation of Examples 3.2 and 4.6). We illustrate the last result on the running example, in which the controllability and observability matrix in (3) are such that $\operatorname{rank} \mathcal{C}=4$ and $\operatorname{rank} \mathcal{O}=2$. The $\mathrm{ABCD}$ form is thus controllable but not observable, and hence non-minimal.

A minimal representation can be derived as follows; here we use the subscript $m$ to index quantities in the minimal state space representation. Write $C$ as $C=C_{m} v^{\prime}$, where

$$
C_{m}=\left(\begin{array}{cc}
1 & 1 \\
-\frac{1}{2} & 1
\end{array}\right), \quad v^{\prime}=\left(\begin{array}{llll}
1 & 1 & 0 & 0 \\
0 & 0 & 1 & 1
\end{array}\right)
$$


and observe that $v^{\prime} A=A_{m} v^{\prime}$, where $A_{m}=\left(\begin{array}{cc}\frac{1}{2} & 0 \\ 0 & 1\end{array}\right)$. Define $x_{m, t}=v^{\prime} x_{t}$ and $B_{m}=v^{\prime} B$, pre-multiply the state equation by $v^{\prime}$ and write the system as

$$
\begin{aligned}
x_{m, t} & =A_{m} x_{m, t-1}+B_{m} w_{t} \\
y_{t} & =C_{m} x_{m, t-1}+D w_{t} .
\end{aligned}
$$

This system is minimal; in fact the controllability and observability matrices of this system satisfy rank $\mathcal{C}_{m}=\operatorname{rank} \mathcal{O}_{m}=2$. Obviously, $C A^{j} B=C_{m} v^{\prime} A^{j} B=C_{m} A_{m}^{j} v^{\prime} B=$ $C_{m} A_{m}^{j} B_{m}$ and hence the so-called Markov coefficients of the two systems are equal.

We next apply Proposition 5.1 to this square minimal ABCD form. The eigenvalues of $F_{m}$ are $\{3 / 5,0\}$; hence they are all stable, and the poor man's invertibility condition correctly signals that $y_{t}$ has an infinite order VAR representation. The unimodularity condition does not apply, because $F_{m}$ has one nonzero eigenvalue. This correctly signals that the system does not have a finite order VAR representation.

\section{Conclusions}

Standard solution methods of DSGE models do not necessarily deliver minimal state space forms. When the ABCD form is non-minimal the poor man's invertibility condition in Fernández-Villaverde et al. (2007) and the unimodularity condition in Ravenna (2007) are not necessary for the existence of a VAR representation of the observables.

In this paper we have presented a check that is valid in the general square case, and hence can be applied to minimal and non-minimal ABCD forms. This illustrates that it is possible to have unstable eigenvalues in the state matrix and in the associate state matrix together with a VAR representation; this is possible because there may exist irrelevant unstable states that are cancelled in the VAR representation of the observables. Finally we show that if the state space form is minimal, the orthogonality condition coincides with the poor man's invertibility condition and the unimodularity condition, because irrelevant states cannot exist.

The present results show that invertibility of linearized DSGE models should be checked either using the present orthogonality conditions or by first transforming the state space form to a minimal representation and then by applying the poor man's invertibility condition to the minimal state space form. Similarly, existence of a finite VAR representation should be checked either using the present orthogonality conditions or by first transforming the state space form to a minimal representation and then by applying the unimodularity condition to the minimal state space form.

Acknowledgments The paper previously circulated with the title "On ABCs (and Ds) of VAR representations of DSGE models". We thank, without implications, the following people for discussions and useful comments on previous versions of the paper: Andrea Attar, Søren Johansen, Marco Lippi, Daniele Massacci, Franco Peracchi, Frank Schorfheide, and James Stock as well as seminar participants. Financial support from the following institutions and grants is is gratefully acknowledged: Einaudi Institute for Economics and Finance (EIEF), Italian MUR Prin Project 2010J3LZEN, University of Rome "La Sapienza" and University of Insubria. 


\section{Appendix: Proofs}

Proof of Proposition 3.1. Observe that $A(0)=T(0)=I_{n_{y}}$. (Sufficiency.) By definition. (Necessity.) If (4) holds, then $w_{t}=M^{-1} A(L) y_{t}$ and hence $T(L) y_{t}=$ $D M^{-1} A(L) y_{t}$, which implies $M=D$ and $T(z)=A(z)$.

Proof of Proposition 3.3. If $F$ is stable, then $(I-F z)^{-1}$ is regular and so is $T(z)$; then apply Proposition 3.1. If $F$ is nilpotent, then $I-F z$ is unimodular, $(I-F z)^{-1}$ is a matrix polynomial and so is $T(z)$; then apply Proposition 3.1.

Proof of Lemma 4.1. Consider (5) and (6); if $C P_{u}(z) B=0$ then $C(I-F z)^{-1} B$ has no pole at $z=\lambda_{u}^{-1}$ and hence the same holds for $T(z)$. Conversely, assume $T(z)$ has no pole at $z=\lambda_{u}^{-1}$; then the same holds for $C(I-F z)^{-1} B$ and thus $C P_{u}(z) B=0$. Because $C P_{u}(z) B=\sum_{j=1}^{m_{u}} \frac{C P_{\lambda_{u}, m_{u}-j} B}{\left(1-\lambda_{u} z\right)^{j}}$, one has $C P_{u}(z) B=0$ if and only if $C P_{\lambda_{u}, m_{u}-j} B$ for $j=1, \ldots, m_{u}$.

Next we show that if the orthogonality condition holds, then $\lambda_{u}$ is also an eigenvalue of $A$. If the orthogonality condition holds, $C P_{\lambda_{u}, 0} B=0$. Write $I-F z=(I-$ $\left.F \lambda_{u}^{-1}\right)+\left(1-\lambda_{u} z\right) \lambda_{u}^{-1} F$ and $(6)$ as $(I-F z)^{-1}=P_{u}(z)+P_{-u}(z)$, where $P_{-u}(z)=$ $\sum_{v=1, v \neq u}^{q} P_{v}(z)+H(z)$; then $(I-F z)(I-F z)^{-1}=I$ implies

$$
\left(I-F \lambda_{u}^{-1}\right) P_{u}(z)+\left(I-F \lambda_{u}^{-1}\right) P_{-u}(z)+\left(1-\lambda_{u} z\right) \lambda_{u}^{-1} F(I-F z)^{-1}=I .
$$

Consider the first term $\left(I-F \lambda_{u}^{-1}\right) P_{u}(z)$ and substitute $P_{u}(z)=\sum_{j=1}^{m_{u}} \frac{P_{\lambda_{u}, m_{u}-j}}{\left(1-\lambda_{u} z\right)^{j}}$; one finds

$$
\left(I-F \lambda_{u}^{-1}\right) P_{u}(z)=\frac{\left(I-F \lambda_{u}^{-1}\right) P_{\lambda_{u}, 0}}{\left(1-\lambda_{u} z\right)^{m_{u}}}+\sum_{j=1}^{m_{u}-1} \frac{\left(I-F \lambda_{u}^{-1}\right) P_{\lambda_{u}, m_{u}-j}}{\left(1-\lambda_{u} z\right)^{j}}
$$

Because $\left(I-F \lambda_{u}^{-1}\right) P_{\lambda_{u}, 0}$ is the only term in (8) that loads $\left(1-\lambda_{u} z\right)^{-m_{u}}$, then (8) implies $\left(I-F \lambda_{u}^{-1}\right) P_{\lambda_{u}, 0}=0$. Similarly, starting from $(I-F z)^{-1}(I-F z)=I$ one finds that $P_{\lambda_{u}, 0}\left(I-F \lambda_{u}^{-1}\right)=0$. Hence $\left(I-F \lambda_{u}^{-1}\right) P_{\lambda_{u}, 0}=P_{\lambda_{u}, 0}\left(I-F \lambda_{u}^{-1}\right)=0$, where $P_{\lambda_{u}, 0} \neq 0$. Because $\lambda_{u}$ is an eigenvalue of $F$, one can write $F-\lambda_{u} I=\alpha \beta^{\prime}$, where $\alpha, \beta$ are $n_{x} \times r$ full column rank matrices, and $r=\operatorname{rank}\left(F-\lambda_{u} I\right)<n_{x}$; moreover, because $\lambda_{u} \neq 0$, one finds $I-F \lambda_{u}^{-1}=-\lambda_{u}^{-1} \alpha \beta^{\prime}$. One then has $P_{\lambda_{u}, 0}=$ $\beta_{\perp} \varphi \alpha_{\perp}^{\prime} \neq 0$, where $\alpha_{\perp}, \beta_{\perp}$ are bases of the orthogonal complements of the column spaces spanned by $\alpha, \beta$ and $\varphi \neq 0$, and hence $C P_{\lambda_{u}, 0} B=C \beta_{\perp} \varphi \alpha_{\perp}^{\prime} B=0$. Because $\operatorname{rank}\left(C \beta_{\perp}\right)=\operatorname{rank}\left(\alpha_{\perp}^{\prime} B\right)=n_{x}-r$ imply $\varphi=0$, it must be that $\operatorname{rank}\left(C \beta_{\perp}\right)<n_{x}-r$ and/or $\operatorname{rank}\left(\alpha_{\perp}^{\prime} B\right)<n_{x}-r$. Next use the projection identities $I=\alpha \bar{\alpha}^{\prime}+\bar{\alpha}_{\perp} \alpha_{\perp}^{\prime}=$ $\bar{\beta} \beta^{\prime}+\beta_{\perp} \bar{\beta}_{\perp}^{\prime}$, where $\bar{a}=a\left(a^{\prime} a\right)^{-1}$, and write

$$
\begin{aligned}
A-\lambda_{u} I & =F-\lambda_{u} I+B D^{-1} C=\alpha \beta^{\prime}+B D^{-1} C=\left(\begin{array}{cc}
\alpha & B
\end{array}\right)\left(\begin{array}{cc}
I_{r} & 0 \\
0 & D^{-1}
\end{array}\right)\left(\begin{array}{l}
\beta^{\prime} \\
C
\end{array}\right) \\
& =\left(\alpha \bar{\alpha}_{\perp}\right)\left(\begin{array}{cc}
I_{r} & \bar{\alpha}^{\prime} B \\
0 & \alpha_{\perp}^{\prime} B
\end{array}\right)\left(\begin{array}{cc}
I_{r} & 0 \\
0 & D^{-1}
\end{array}\right)\left(\begin{array}{cc}
I_{r} & 0 \\
C \bar{\beta} & C \beta_{\perp}
\end{array}\right)\left(\begin{array}{c}
\beta^{\prime} \\
\bar{\beta}_{\perp}^{\prime}
\end{array}\right) .
\end{aligned}
$$


Because $\operatorname{rank}\left(C \beta_{\perp}\right)<n_{x}-r$ or $\operatorname{rank}\left(\alpha_{\perp}^{\prime} B\right)<n_{x}-r$, one has that $A-\lambda_{u} I$ is singular; this shows that $\lambda_{u}$ is an eigenvalue of $A$.

Proof of Propositions 4.2 and 4.3. $T(z)$ is regular if and only if $C P_{u}(z) B=0$ for all unstable eigenvalues and it is a matrix polynomial if and only if $C P_{u}(z) B=0$ for all eigenvalues. The statement then follows from Proposition 3.1 and Lemma 4.1.

Proof of Proposition 4.4. (Sufficiency.) If the orthogonality condition holds at $\lambda_{u}$, then $C P_{\lambda_{u}, 0} B=0$; because $C P_{\lambda_{u}, 0} B=C \beta_{\perp} \varphi \alpha_{\perp}^{\prime} B$ and $\varphi \neq 0$, it follows that $\operatorname{rank}\left(C \beta_{\perp}\right)<n_{x}-r$ and/or $\operatorname{rank}\left(\alpha_{\perp}^{\prime} B\right)<n_{x}-r$, see the proof of Lemma 4.1. (Necessity.) We first show that if $\operatorname{rank}\left(C \beta_{\perp}\right)<n_{x}-r$ and/or $\operatorname{rank}\left(\alpha_{\perp}^{\prime} B\right)<n_{x}-r$, then $\lambda_{u}$ is non-controllable and/or non-observable; we then apply Kalman's decomposition theorem for non-controllable and/or non-observable linear systems.

For the PBH rank test, see e.g. Kailath (1980, Theorem 6.2.6), one has that $\lambda_{u}$ is controllable if and only if $\operatorname{rank}\left(A-\lambda_{u} I: B\right)=n_{x}$ and it is observable if and only if $\operatorname{rank}\left(A^{\prime}-\lambda_{u} I: C^{\prime}\right)=n_{x}$. Observe that $\operatorname{rank}\left(A-\lambda_{u} I: B\right)=\operatorname{rank}\left(F-\lambda_{u} I: B D^{-1}\right)$; in fact

$$
\left(\begin{array}{cc}
A-\lambda_{u} I & B
\end{array}\right)\left(\begin{array}{cc}
I_{n_{x}} & 0 \\
-D^{-1} C & D^{-1}
\end{array}\right)=\left(\begin{array}{cc}
F-\lambda_{u} I & B D^{-1}
\end{array}\right)
$$

and similarly one finds that $\operatorname{rank}\left(A^{\prime}-\lambda_{u} I: C^{\prime}\right)=\operatorname{rank}\left(F^{\prime}-\lambda_{u} I:-C^{\prime} D^{-1 \prime}\right)$. Recall that $F-\lambda_{u} I=\alpha \beta^{\prime}$ and use the projection identities $I=\alpha \bar{\alpha}^{\prime}+\bar{\alpha}_{\perp} \alpha_{\perp}^{\prime}=\bar{\beta} \beta^{\prime}+\beta_{\perp} \bar{\beta}_{\perp}^{\prime}$ to write

$$
\begin{aligned}
\left(\begin{array}{cc}
F-\lambda_{u} I & B D^{-1}
\end{array}\right) & =\left(\begin{array}{cc}
\alpha \beta^{\prime} \alpha \bar{\alpha}^{\prime} B D^{-1}+\bar{\alpha}_{\perp} \alpha_{\perp}^{\prime} B D^{-1}
\end{array}\right) \\
& =\left(\begin{array}{cc}
\alpha \bar{\alpha}_{\perp}
\end{array}\right)\left(\begin{array}{cc}
\beta^{\prime} & \bar{\alpha}^{\prime} B D^{-1} \\
0 & \alpha_{\perp}^{\prime} B D^{-1}
\end{array}\right), \\
\left(\begin{array}{c}
F-\lambda_{u} I \\
-D^{-1} C
\end{array}\right) & =\left(\begin{array}{c}
\alpha \beta^{\prime} \\
-D^{-1} C \bar{\beta} \beta^{\prime}-D^{-1} C \beta_{\perp} \bar{\beta}_{\perp}^{\prime}
\end{array}\right) \\
& =\left(\begin{array}{cc}
\alpha & 0 \\
-D^{-1} C \bar{\beta} & -D^{-1} C \beta_{\perp}
\end{array}\right)\left(\begin{array}{c}
\beta^{\prime} \\
\bar{\beta}_{\perp}^{\prime}
\end{array}\right) .
\end{aligned}
$$

Similar expressions hold replacing $F, B D^{-1},-D^{-1} C$ with $A, B, C$. This shows that $\lambda_{u}$ is non-controllable and/or non-observable, both for the original ABCD form (1) and for the associate state space (2).

We next apply Kalman's decomposition theorem, see e.g. Theorem 6.6 in Antsaklis and Michel (2007, p. 245), to the associate state space (2), with $(A, B, C, D)$ replaced by $\left(F, G, H, D^{-1}\right), G=B D^{-1}, H=-D^{-1} C$. From Antsaklis and Michel (eq. (7.6) 2007 , p. 278), one has that the transfer function $D^{-1} T(z)$ has representation $D^{-1}+H_{1}\left(I-F_{11} z\right)^{-1} G_{1} z$ where $F_{11}$ has eigenvalues that are both controllable and observable, and $H_{1}, G_{1}$ are constructed as $C_{1}, B_{1}$ in eq. (7.6) of Antsaklis and Michel (2007). This implies that $T\left(\lambda_{u}^{-1}\right)$ is finite, and hence that $C P_{u}(z) B=0$ by Lemma 4.1 .

Proof of Proposition 4.5. Direct consequence of Propositions 4.3 and 4.4. 
Proof of Proposition 5.1. (Sufficiency.) See proof of Proposition 3.3. (Necessity.) For the PBH rank test, see e.g. Kailath (1980, Theorem 6.2.6), one has that if the ABCD form is minimal then $\operatorname{rank}(A-\lambda I: B)=\operatorname{rank}\left(A^{\prime}-\lambda I: C^{\prime}\right)=n_{x}$ for all $\lambda \in \mathbb{C}$. This implies, see the proof of Proposition 4.4, that $\operatorname{rank}\left(C \beta_{\perp}\right)=\operatorname{rank}\left(\alpha_{\perp}^{\prime} B\right)=n_{x}-r$; hence $C P_{\lambda_{u}, 0} B=C \beta_{\perp} \varphi \alpha_{\perp}^{\prime} B=0$ contradicts $\varphi \neq 0$ and thus the orthogonality condition cannot hold. This implies that $T(z)$ is regular only if $F$ is stable and it is a matrix polynomial only if $F$ is nilpotent. Finally apply Proposition 3.1 .

\section{References}

An, S., \& Schorfheide, F. (2007). Bayesian analysis of DSGE models. Econometric Reviews, 26, 113-172. Antsaklis, P., \& Michel, A. (2007). A linear systems primer. Basel-Boston-Berlin: Birkhäuser.

Bart, H., Gohberg, I., Kaashoek, M., \& Ran, A. (2008). Factorization of matrix and operator functions: The state space method. Basel-Boston-Berlin: Birkhäuser.

Binder, M., \& Pesaran, H. (1997). Multivariate linear rational expectations models. Econometric Theory, $13,877-888$.

Brockwell, P., \& Davis, R. (1987). Time series: Theory and methods. Berlin: Springer.

Chang, Y., Doh, T., \& Schorfheide, F. (2007). Non-stationary hours in a DSGE model. Journal of Money, Credit and Banking, 39, 1357-1373.

Chari, V., Kehoe, P., \& McGrattan, E. (2005). A critique of structural VARs using real business cycle theory. Working Papers 631, Federal Reserve Bank of Minneapolis.

Christiano, L., Eichenbaum, M., \& Evans, L. (2005). Nominal rigidities and the dynamic effects of a shock to monetary policy. Journal of Political Economy, 113, 1-45.

Christiano, L. J., Eichenbaum, M., \& Vigfusson, R. (2006). Assessing structural VARs. In D. Acemoglu, K. Rogoff, \& M. Woodford (Eds.), NBER macroeconomics annual 2006 (Vol. 21, pp. 1-106). Cambridge, MA: The MIT Press.

Fernández-Villaverde, J., Rubio-Ramírez, J., Sargent, T., \& Watson, M. (2007). ABCs (and Ds) of understanding VARs. American Economic Review, 97, 1021-26.

Fischer, W., \& Lieb, I. (2012). A course in complex analysis: From basic results to advanced topics. New York: Vieweg+Teubner Verlag.

Franchi, M. (2013). Comment on: Ravenna, F., 2007. Vector autoregressions and reduced form representations of DSGE models. Journal of Monetary Economics, 54, 2048-2064. Working paper DSS-E3 2, Sapienza University of Rome.

Franchi, M., \& Paruolo, P. (2011a). A characterization of vector autoregressive processes with common cyclical features. Journal of Econometrics, 163, 105-117.

Franchi, M., \& Paruolo, P. (2011b). Inversion of regular analytic matrix functions: Local Smith form and subspace duality. Linear Algebra and its Applications, 435, 2896-2912.

Franchi, M., \& Vidotto, A. (2013). A check for finite order VAR representations of DSGE models. Economics Letters, 120, 100-103.

García-Cicco, J., Pancrazi, R., \& Uribe, M. (2010). Real business cycles in emerging countries? The American Economic Review, 100, 2510-2531.

Hannan, E., \& Deistler, M. (1988). The statistical theory of linear systems. New York: Wiley.

Hansen, L., \& Sargent, T. (1980). Formulating and estimating dynamic linear rational expectations models. Journal of Economic Dynamics and Control, 2, 7-46.

Howlett, P. (1982). Input retrieval in finite dimensional linear systems. Journal of Australian Mathematical Society (Series B), 23, 357-82.

Johansen, S. (1988). Statistical analysis of cointegration vectors. Journal of Economic Dynamics and Control, 12, 231-254.

Johansen, S. (1996). Likelihood-based inference in cointegrated vector auto-regressive models. Oxford: Oxford University Press.

Johansen, S. (2009). Representation of cointegrated autoregressive process with application to fractional process. Econometric Reviews, 28, 121-145.

Kailath, T. (1980). Linear systems. Englewood Cliffs, NJ: Prentice Hall. 
Kapetanios, G., Pagan, A., \& Scott, A. (2007). Making a match: Combining theory and evidence in policyoriented macroeconomic modeling. Journal of Econometrics, 136, 565-594.

Klein, P. (2000). Using the generalized Schur form to solve a multivariate linear rational expectations model. Journal of Economic Dynamics and Control, 24(10), 1405-1423.

Komunjer, I., \& Ng, S. (2011). Dynamic identification of dynamic stochastic general equilibrium models. Econometrica, 79, 1995-2032.

Kurmann, A., \& Otrok, C. (2011). News shocks and the term structure of interest rates: A challenge for DSGE models. Working Paper.

Leeper, E. M., Walker, T. B., \& Yang, S.-C. S. (2013). Fiscal foresight and information flows. Econometrica, 81(3), 1115-1145. doi:10.3982/ECTA8337.

Lippi, M., \& Reichlin, L. (1993). The dynamic effects of aggregate demand and supply: Comment. American Economic Review, 83, 644-652.

Lippi, M., \& Reichlin, L. (1994). Var analysis, non-fundamental representations, Blaschke matrices. Journal of Econometrics, 63, 307-325.

Ravenna, F. (2007). Vector autoregressions and reduced form representations of DSGE models. Journal of Monetary Economics, 54, 2048-2064.

Rosenbrock, H. (1970). State-space and mltivariable theory. London: Thomas Nelson and Sons.

Schmitt-Grohé, S. (2010). Comment on: Letting different views about business cycles compete. NBER Macroeconomics Annual, 24, 475-490.

Sims, C. A. (2001). Solving linear rational expectations models. Computational Economics, 20(1-2), 1-20.

Sims, E. (2012). News, non-invertibility, and structural VARs. Advances in Econometrics, 28, 81-135.

Smets, F., \& Wouters, R. (2007). Shocks and frictions in US business cycles: A Bayesian DSGE approach. The American Economic Review, 97, 586-606.

Uhlig, H. (1999). A toolkit for analyzing nonlinear dynamic stochastic models easily. In R. Marimon \& A. Scott (Eds.), Computational methods for the study of dynamic economies (pp. 30-61). Oxford: Oxford University Press. 